\title{
DERECHO Y MORAL: VINDICACIÓN DEL OBSERVADOR CRÍTICO Y APORÍA DEL IUSNATURALISTA*
}

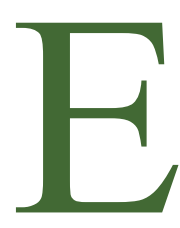

$\mathrm{n}$ el encuentro alicantino entre colegas españoles y escandinavos que se recoge en este número y que fue, por cierto, tan intenso y fecundo como amistoso y distendido, el tema de la separación o conexión entre derecho y moral fue, como no podía ser menos, el que suscitó debates más apasionados. Mi propuesta de entonces quiso poner de manifiesto que aquellos iusnaturalistas que mantienen sin paliativos esa conexión pueden verse comprometidos con una visión autoritaria de la moral, y ello no por simples vicisitudes históricas o coyunturales, sino por más profundas razones de carácter teórico. Ahora bien, como la cuestión de las relaciones entre moral y derecho es un sendero innumerable, no advertí a tiempo que me proponía hablar de una de las estaciones intermedias de uno de los itinerarios sin haber hecho ni siquiera mención de las estaciones anteriores o de los demás itinerarios. El grueso de nuestras discusiones, como suele suceder en este tema, se centró en una encrucijada que se encuentra situada mucho antes del asunto que yo proponía: la encrucijada de los «puntos de vista». Tendré, por tanto, que hacer una tentativa de explicar cómo se puede pasar por ella para arribar al tema del iusnaturalismo y sus apuros.

Creo que podemos estar de acuerdo en que el punto de partida de toda la cuestión derecho-moral podría ser la pregunta

* El presente trabajo se ha beneficiado no sólo de las vibrantes discusiones del Seminario de Alicante, sino de largas y exhaustivas conversaciones con Juan Carlos Bayón y Alfonso Ruiz Miguel y de una extensa epístola académica que me envió desde Oxford Juan Ruiz Manero. Y se ha beneficiado tanto que aquellos que sólo conocieron el borrador inicial dudarán de emparentarlo con éste. Las mejoras que haya experimentado respecto de aquél se deben a sus matizaciones y sus abiertas críticas, y son tantas que prefiero reconocerlas de golpe y no párrafo por párrafo, lo que sería premioso. De los errores que sin duda subsisten soy yo el único responsable. 
por el sentido y el alcance de este enunciado: «Esto es derecho válido pero es inmoral.» En principio se puede decir que quienes afirman la conexión necesaria entre derecho y moral piensan que en el enunciado en cuestión hay algún tipo de contradicción, mientras que aquellos que niegan tal conexión no ven en el mismo contradicción alguna. Pero, claro, para saber si hay o no hay contradicción tendremos que determinar antes qué significa ese enunciado. Y ahí es precisamente donde surge la tupida red de itinerarios. Porque el aserto «esto es derecho válido pero es inmoral» es extremadamente complejo de entender. No significará lo mismo si lo emite un observador externo que si lo emite un insider, es decir, un participante identificado con ese derecho. Cambiará también si por «Esto» entendemos un ordenamiento concebido como un todo o una norma aislada. Su alcance dependerá a su vez de que «válido» signifique perteneciente al sistema, vigente empíricamente o vinculante. $\mathrm{Y}$ variará, por último, según hablemos de moralidad positiva o de moralidad crítica. Si tenemos en cuenta todas esas variables juntas el enunciado en cuestión presenta veinticuatro significados posibles. Un buen número de itinerarios, por tanto. Y no son demasiados si se piensa que ha habido quien ha sugerido la posibilidad de que sean nada menos que sesenta y cuatro (Alexy, 1989). Así que, con el fin de evitar en lo posible discusiones estériles anuncio que me refiero solamente a uno de ellos: aquel en el que dicho enunciado es emitido por un observador externo sobre una norma aislada que satisface los criterios de pertenencia a un sistema y es vigente empíricamente pero, para las pautas de moralidad crítica de ese observador, es inmoral. En este significado decir «Esto es derecho válido pero es inmoral» no es en absoluto contradictorio y, por tanto, en este significado no hay una conexión necesaria entre derecho y moral.

Ahora bien, ¿por qué hacer una opción de significado como ésta? ¿Cuál puede ser la razón que aconseje en este tema resistir la creciente presencia del «punto de vista interno» y volver a recordar algunas de las virtudes heurísticas de los enunciados externos? Mi respuesta es que tal elección puede apoyarse en una correcta delimitación del problema concreto que se pretende enfrentar con ella. Porque seguramente la cuestión Derecho-Moral no es, como parece, una sola cuestión, sino una compleja trama de problemas lo suficientemente heterogéneos. Y, de entre 
ellos, el problema particular que creo merece ser subrayado una vez más es un problema práctico: Nos tropezamos con frecuencia con normas que han pasado todos los controles formales de pertenencia a un sistema jurídico y que son, además, aceptadas y aplicadas oficialmente como tales, pero que, sin embargo, violan flagrantemente exigencias morales o demandas de justicia ampliamente compartidas. Normas, por ejemplo, que vulneran claramente la Declaración Universal de Derechos Humanos. Tales normas son consideradas jurídicas pero son tachadas de injustas o inmorales; incluso por la misma persona. De hecho se organizan con frecuencia campañas destinadas a lograr su «derogación», y si tiene sentido tratar de derogar una norma es porque esa norma está de algún modo en vigor como jurídica. Decir de ella que «es derecho válido pero es inmoral» resulta un enunciado perfectamente idóneo a esos propósitos prácticos. Creo, además, que dichos propósitos están muy presentes en la historia misma del problema, que surge seguramente como problema frente a situaciones en las que de lo que se trataba era de poner en pie una plataforma para enfrentar el derecho empírico con pautas de moralidad crítica no reconocidas ni incorporadas a él. Lo que, desde el siglo XVIII, los ilustrados trataron de defender es la posibilidad de actitudes críticas frente al poder político y la legislación vigente, y para hacer sitio a esa posibilidad fue para lo que advirtieron que el derecho podía ser inmoral y que decirlo con toda claridad no constituía ninguna contradicción en los términos. No me parece, por ello, superfluo vindicar una vez más a un observador externo capaz de dar sentido a una denuncia que se me antoja extremadamente educadora y necesaria. Al fin y al cabo, aunque todo el mundo viva en el seno de algún ordenamiento jurídico, la mayoría lo hace en el papel de sujeto paciente o, lo que es lo mismo, de mero observador extraño que sufre o disfruta como destinatario la regulación jurídica en cuestión. Animarle a que ose decir: «Muy bien, eso será derecho, pero es una perfecta inmoralidad», me parece aceptar un legado de la Ilustración que merece ser transmitido incesantemente.

Ahora bien, esa elección de un significado tan particular para ese enunciado, podría ser, quizás, sometida a crítica desde el punto de vista teórico o metodológico. ¿No serán, al fin y al cabo, los observadores externos sujetos irremediablemente incapacitados para conocer lo que el derecho válido es? Dedicaré algunas páginas a argumentar en favor de cada uno de los sentidos en que interpreto los términos del enunciado en cuestión para poder llegar a la conclusión de que ese interrogante merece una respuesta negativa. 
1. En primer lugar está, por supuesto, la gran cuestión del punto de vista interno y el punto de vista externo. La polarización de la teoría entre ambos se ha visto a veces alimentada por una estrategia argumental que tendía a «esperpentizar» al observador externo dibujándolo como una suerte de alienígena incapaz de tener en cuenta las relaciones de los seres humanos con sus pautas de comportamiento; una mente de una ajeneidad total que sólo es capaz de registrar regularidades estadísticas exteriores en las conductas de su «objeto» de observación. Pero como es de sobra conocido, el observador externo no tiene por qué ser tan pintorescamente ajeno. Puede, desde fuera, dar cuenta de la aceptación de las normas como razones para la acción y pautas de crítica. Puede incluso expresarse a través de lo que Kelsen denominó «proposiciones normativas» o a través de que lo que Raz llama «enunciados jurídicos distanciados». Puede, creo, por tanto, hablar con todo sentido de «derecho válido».

Veo, además, en el «punto de vista interno» algunos posibles problemas. Cuando se le presenta como la opción epistemológica correcta puede conducir a un extremado relativismo. MacCormik ha recordado recientemente que la validez de las normas es algo relativo a los sistemas jurídicos. Una norma es válida de acuerdo a los criterios internos de un sistema y no es válida en otro sistema (MacCormik, 1987). Pero una cosa es la validez de una norma y otra distinta la validez del conocimiento de que una norma es válida. Si al llamado «punto de vista interno» se le atribuye también el ser la única manera precisa y exhaustiva de conocer qué es derecho válido entonces esto obliga a defender una forma severa de relativismo epistemológico en materias jurídicas $\mathrm{y}$, temo, en todo lo referente a prácticas sociales en general. Piénsese que lo que se afirma en este caso es que el valor de verdad de una proposición sobre la validez de las normas es relativo a la posición del sujeto cognoscente. Si no se puede conocer lo que es una norma o una práctica social cuando no se es un «insider», entonces los criterios de comprensión de una práctica $\mathrm{y}$, en definitiva, de la verdad de las proposiciones que dan cuenta de ella, serán también internos a dicha práctica. La intersubjetividad de esos juicios sólo podría darse entre los identificados con esa práctica y, en nuestro caso, con el sistema jurídico. Pues bien, aunque no soy partidario de rechazar un cierto tipo de relativismo gnoseológico moderado, creo, sin embargo, que el tipo de relativismo a que nos obliga esta perspectiva es inaceptable. Me parece que sobre la verdad del enunciado «La norma A es derecho válido en el sistema $\mathrm{X} »$, entre el punto de vista de un 
observador mínimamente informado y el del identificado con el sistema tiene que haber coincidencia porque su posición ante ese sistema es irrelevante a efectos cognitivos. Otra cosa, claro está, es que esa posición tenga otras implicaciones.

Otro foco de interrogantes que suscita el punto de vista interno proviene de la naturaleza misma del problema de la noción de derecho válido, que es la clave de la cuestión derecho/moral. Hart insiste una y otra vez en que esta cuestión es «conceptual»o «definicional». Y es evidente que su clave implícita, es decir, el problema de qué sea «derecho válido», es una pura cuestión definicional. Ahora bien, desde un punto de vista lógico las definiciones son enunciados metalingüísticos, es decir, enunciados que pertenecen a un dominio de segundo orden respecto del orden que constituye el lenguaje-objeto. Y las definiciones en el derecho tampoco se ubican lógicamente en el plano de las normas jurídicas sino en un metalenguaje que habla del lenguaje de las normas jurídicas. Esto se advierte por ejemplo en la teoría de Hart, de acuerdo con la cual el tipo de «norma secundaria»y, en particular, la regla de reconocimiento, es una «norma sobre normas» es decir, un enunciado metanormativo. Incluso se ha afirmado (Bulygin, 1976) que tal regla de reconocimiento es, en realidad, una regla conceptual, una definición. Si esto es correcto, entonces el «punto de vista interno» tiene que vérselas con órdenes distintos de lenguajes, el de las definiciones y el de las normas en sentido estricto. ¿Cómo lo hace? Seguramente está claro qué significa hablar «desde dentro» en relación con las normas jurídicas de conducta. Pero cuando se desliza uno de ese lenguaje al metalenguaje de las definiciones, entonces, con respecto a aquél, ya no se están emitiendo «enunciados internos» sino enunciados externos sobre aquellas normas de conducta. Los «internal statements» se dan cuando se usan las normas de conducta, pero no cuando se definen. Cuando se definen se está situado en un nivel metalingüístico que no puede ser, por lógica, interno al lenguaje-objeto. El «insider», por tanto, «sale» del lenguaje de las normas de conducta cuando apela a la regla de reconocimiento. Es, con respecto a aquél, también un «observador externo». Pero, ¿y con respecto a la propia regla de reconocimiento? Si aceptamos, siguiendo a Bulygin, que se trata de una definición, el «insider» la usará normativamente «desde dentro», mientras que el espectador lo hará sólo lexicográficamente. Es decir, mientras que el identificado con ella no la usará sólo como criterio cognoscitivo sino también como premisa normativa en un hipotético razonamiento práctico, el observador hará uso de ella sólo como instrumento para describir exhaustivamente una 
práctica. Lo importante, sin embargo, es que el resultado definicional es equivalente en ambos casos.

Hay un tercer problema recordado con frecuencia y que se plantea también a quienes mantienen la perspectiva del «punto de vista interno». Cuando se dice que no podemos dar cuenta de lo que el derecho es sino mediante «enunciados internos» puede incurrirse en una petición de principio. Sólo puede hablarse de derecho válido -se nos dice- si al menos los funcionarios oficiales tienen, respecto de las normas en cuestión, esa actitud interna, lo que para ciertas versiones fuertes de esta perspectiva (Nino, 1989) significa que consideran a esas normas como moralmente vinculantes. Es esto, precisamente, lo que establece la conexión conceptual entre derecho y moral. Ahora bien, para aplicar este criterio cognoscitivo tenemos que saber antes qué cosa es o quién es un «official», es decir, un funcionario, un juez, un legislador, etc... Y la única manera posible de saber esto es apelando a normas jurídicas, a las normas jurídicas que confieren poderes. Estas normas jurídicas son también derecho válido, con lo que al introducir en el definiens de «derecho válido» a los funcionarios y jueces el argumento se hace circular. Es una circularidad de la que además, no es fácil salir «desde el punto de vista interno» porque ¿qué respuesta podría darse a la pregunta sobre la validez de las normas que apoderan a los «officials»? ¿Qué los propios «officials» tienen hacia ellas una actitud interna? Sería un poco grotesco suponer que los jueces son jueces porque se consideran a sí mismos moralmente jueces. Y si esto no es así, entonces no sólo serían los jueces y funcionarios los que habrían de tener dicha actitud interna, sino también una parte de los ciudadanos, que deberían mirar a sus autoridades «internamente» como autoridades morales, con lo que el derecho acabaría por ser equivalente a una moralidad positiva muy institucionalizada.

Me parece que la cuestión es, más bien, que sabemos lo que el derecho es, no porque sepamos cuál es la actitud de los funcionarios, sino porque sabemos quiénes son los funcionarios. La gente (también los funcionarios) suele tener una similar actitud interna hacia muchos tipos de reglas y pautas y si podemos distinguir entre ellas las que son jurídicas de las que no lo son es porque tenemos un criterio para hacerlo al margen de aquella actitud. Ese es el criterio que nos permite identificar al oficial, legislador y juez como tal funcionario, el criterio que define lo que es el derecho. Y creo que tal criterio es expresable básicamente en términos externos de eficacia general, o para usar terminología hartiana, de existencia de hecho de una práctica com- 
pleja de aplicar la regla de reconocimiento (Silvana Castiglione, 1983). Pero como digo, tal existencia de hecho se puede expresar perfectamente con un enunciado externo.

Todas estas consideraciones nos llevan, en suma, a la conclusión de que, por lo que respecta a la competencia epistemológica para determinar qué es «derecho válido», el observador externo se encuentra tan capacitado como el «insider» para llevar dicha tarea a cabo. Un poco más adelante surgirá, sin embargo, otro problema emparentado con esto.

2. La elección del enfoque «norma a norma» en lugar de optar por una visión global del derecho «como un todo» es relativamente sencilla de justificar. Es extremadamente difícil hacer pronunciamientos morales sobre todo un sistema jurídico y, en el caso de que tal cosa pudiera hacerse sin excesiva osadía es muy posible que tales pronunciamientos morales fueran en la mayoría de los casos (aunque no necesariamente en todos) una función de pronunciamientos morales sobre normas concretas pertenecientes al todo. Si un orden jurídico es injusto lo es seguramente porque muchas de las normas que pertenecen a él lo son. (Sobre otros problemas de esta distinción, Laporta, 1990).

3. Más incógnitas puede suscitar la elección del significado desde una noción de validez como pertenencia formal al sistema. Aquí, inopinadamente, vuelve a aparecer el tema de los puntos de vista. Uno de mis interlocutores (Ruiz Manero) sugiere, creo que con razón, que no es tan claro que la validez como pertenencia de una norma al sistema sea tan fácilmente diferenciable de la validez como vinculatoriedad, o, para mejor expresarlo, que no es tan claro que ambas acepciones de «validez», aunque diferenciables, sean conceptualmente independientes puesto que de algún modo la validez como pertenencia es noción tributaria de la validez como vinculatoriedad, porque cuando se dice que una norma pertenece al sistema se está presuponiendo implícitamente que hay ciertos órganos del sistema que tienen hacia ciertos enunciados una actitud interna, lo que quiere decir que antes que la pura pertenencia se da una validez como vinculatoriedad y en la medida en que se da podemos encontrar criterios de pertenencia. $\mathrm{O}$, lo que es lo mismo, que la validez como vinculatoriedad es condición necesaria para la validez como pertenencia. Pero esto, que seguramente es correcto, puede quizás ser formulado de otra manera. Lo que este planteamiento viene a decirnos es que no es tan fácil desintegrar la noción de validez en acepciones plenamente diferenciadas y ajenas entre sí: cada una de ellas (pertenencia, vigencia, vinculatoriedad) sería un aspecto de la misma noción. O sería la misma noción enfocada desde 
distintos «puntos de vista». El punto de vista más decididamente interno y comprometido daría cuenta del aspecto de vinculatoriedad. Un punto de vista distanciado (detached), a mitad del camino, por así decirlo, entre lo interno y lo externo, daría cuenta del aspecto de pertenencia, tanto describiendo la creencia en la vinculatoriedad como usándola a efectos informativos como criterio para determinar qué normas son percibidas como jurídicas y, por tanto, como integrantes del conjunto o sistema jurídico. Y, por fin, un punto de vista más declaradamente externo para reflejar en términos puramente descriptivos la vigencia empírica, es decir, las regularidades externas de conducta. Si esto es correcto (como me lo sugiere Ruiz Miguel), lo importante entonces es que el predicado «válido», cualquiera que sea el enfoque o punto de vista desde el que se atribuya, tiene que ser atribuido a la misma norma o conjunto de normas so pena de caer en desacuerdos incomprensibles. Porque si es verdad que no es posible la existencia de «vigencia empírica» sin compromiso interno de los órganos del sistema, o que no puede haber «proposiciones» de la ciencia jurídica sin algún grado de vinculatoriedad interna de las normas descritas mediante ellas, entonces el referente normativo de todos los «puntos de vista» tiende a ser el mismo. Y también sucede, en sentido contrario, que si es existencialmente imposible que haya un «derecho válido» desde el punto de vista interno si ese «derecho válido» no tiene a su vez una razonable presencia empírica en las conductas de los ciudadanos, ambas perspectivas de «validez» tienen que confluir para señalar a las mismas normas. Lo que con ello quiero concluir, una vez más, es que la idea de validez como pertenencia formal utilizada con funciones descriptivas por un observador externo no es algo cuyos resultados medidos en «unidades» epistemológicas, es decir, cuyos resultados como herramienta de conocimiento, sean distorsionantes, pobres o erróneos. Por el contrario, lo que quiero defender es que son en muy amplia medida coincidentes con los derivados de la idea de validez como vinculatoriedad enfocada desde dentro.

4. Y, por último, queda la elección en favor de la moralidad crítica frente a la moralidad positiva. En cuanto a esto creo que el punto de vista externo es más completo y ambicioso que el interno por la sencilla razón de que es el observador externo (o quien se sitúe en esa posición) el único que puede establecer una dicotomía como ésa, pues es el único que puede describir una práctica moral empírica y contrastar esa práctica con pautas morales críticas, ya que, por definición, no está implicado en ella. Para el insider, por el contrario, esta distinción es difícil de 
establecer porque la moralidad del derecho que practica desde dentro es para él la única moralidad posible. En el momento que la pone en cuestión deja, automáticamente, de ser un insider. Para el problema de las relaciones moral/derecho esta es una consideración muy interesante cuyas implicaciones se pueden ponderar desglosándola en cuatro supuestos.

a) El insider que afirma que hay una conexión necesaria entre derecho y moral lo único que expresa realmente es su propia aceptación de las normas jurídicas como pautas morales válidas y, por tanto, se refiere siempre a la única moralidad posible para él: la moralidad crítica.

b) El insider que afirma que no hay tal conexión necesaria parece una figura incomprensible, de acuerdo con los críticos de Hart (Nino). La mera condición de «insider» implica, para ellos, un compromiso moral, y, por tanto, esta posición sería contradictoria. Hart, como es sabido, se resiste a aceptar este corolario de su propia teoría. Tampoco yo lo veo tan claro.

c) El outsider o espectador que afirma que hay una conexión necesaria entre derecho y moralidad se está refiriendo, naturalmente, a la moralidad positiva, y lo único que nos dice es la trivialidad de que siempre que un orden jurídico existe empíricamente hay alguien que cree moralmente en él. Siempre hay un cierto grado de coincidencia entre las normas jurídicas y las pautas de la moralidad positiva. Cuanto menor es ese grado más inestable es el sistema. Si falta absolutamente el sistema se desvanece.

d) El espectador que afirma que no hay una conexión conceptual entre derecho y moral y se refiere con ello a la moralidad crítica.

De todas estas posiciones la que, desde el punto de vista cognoscitivo, parece más poderosa es la última: Expresa -en primer lugar- una afirmación desde la moralidad crítica; en segundo lugar, no es incompatible con c), ya que puede, a su vez, dar cuenta descriptivamente de una moralidad positiva vinculada al derecho; puede -además- situarse respecto a este problema en la posición de $b$ ) si es que ésta es posible y, por último, aunque no comparte el punto de vista a) también puede dar cuenta descriptivamente de él. Es, por tanto, la que más posibilidades tiene. Lo único que, al parecer, le está vedado es tomar una norma del orden jurídico que observa como premisa moral para un razonamiento práctico, pero esto, a mi juicio, no limita 
su aptitud para explicar lo que ve ni su capacidad de «comprensión» hacia ello. Yo, al menos, no advierto que lo haga.

\section{II}

Una vez aducidas las razones que pueden apoyar una vindicación del punto de vista del observador crítico para afrontar el problema práctico que está en el núcleo de la propuesta de separación conceptual de derecho y moral, conviene pasar a dilucidar qué problema puede encontrar el iusnaturalista que mantiene la conexión mencionada. Entiendo por iusnaturalista, precisamente, a alguien que afirma la existencia de esa conexión necesaria o conceptual y que, además, y simultáneamente, la une a la afirmación de que hay valores o pautas morales objetivos y cognoscibles, de tal forma que si el derecho no incorpora de algún modo dichas pautas o valores no merece, en sentido estricto, la calificación de «derecho», o, lo que es lo mismo, a «eso» no le cuadra el concepto o el nombre de «derecho». ¿Dónde estaría situado este iusnaturalista? Me parece que, necesariamente, tendría que alimentar la pretensión de hablar como un observador externo y objetivo que afirma la conexión conceptual entre derecho y moralidad crítica. En efecto, no le resultaría suficiente hablar en términos puramente empíricos de moralidad meramente positiva puesto que lo que afirma es la validez de ciertas pautas de ética al margen de su vigencia o su no vigencia empírica. El mero reproducir el hecho de que una cierta moralidad positiva coincida con el derecho no es lo que el iusnaturalista pretende. Y tampoco sería para él satisfactorio situarse en el «punto de vista interno» porque no trata simplemente de expresar una actitud interna hacia cualesquiera normas jurídicas, sino de afirmar un imperativo moral anterior a cualquier actitud. Lo que le diferencia del observador externo positivista que está en el supuesto $d$ ) de los anteriormente mencionados es, precisamente, que apelando a una moralidad crítica pretende que hay una conexión conceptual entre el derecho y esa moralidad. Y cuando no la hay, «aquello», obviamente, no puede ser llamado «derecho».

Pues bien, si un jurista así pretendiera identificar ciertas normas como jurídicas mediante una apelación a valores morales como la justicia, la libertad o el bien común, o a normas morales sobre lo que es correcto o incorrecto hacer o decidir, se encontraría inmediatamente frente a una cantidad enorme de acciones, conductas, decisiones, reglas, estados de cosas... etc., que cumplieran con esos requisitos. El criterio sería entonces 
inútil porque es evidente que no se puede mantener que toda acción, decisión, regla, o institución justa es, por ello, también jurídica. Nadie dudará que puede haber más situaciones justas que situaciones jurídicas. En consecuencia, la clase de las acciones, decisiones, etc.... justas no es coextensa con la de las acciones, decisiones, etc.... jurídicas. Le será, por tanto, necesario recurrir a un criterio adicional para diferenciar entre acciones o reglas justas jurídicas y acciones o reglas justas no jurídicas. Nótese, por lo demás, que en esto se encuentra quizás el iusnaturalista en el mismo caso que el «insider», porque éste, que tiene en principio una actitud interna de aceptación moral de muchos tipos de pautas y normas necesitará también un criterio adicional para saber de entre ellas las que son jurídicas y las que no son jurídicas. Sería descabellado suponer que son normas jurídicas todas aquellas hacia las que el «insider» tiene una actitud interna.

Así pues, ambos necesitan de otro criterio complementario del anterior. Y lo más sorprendente de ese criterio es que tiene precisamente como misión identificar la clase de las reglas y decisiones jurídicas y, para ello, ha de articularse en buena medida en forma de definición o, lo que quizás sea equivalente, en forma de regla constitutiva, o, más allá de un simple parentesco verbal, en forma de «norma constituyente», lo que relaciona inmediatamente dicho criterio con los componentes «fundamentales» del universo de lo jurídico. Esto es, desde luego, algo muy paradójico. Resulta que el iusnaturalista propone un criterio fuerte de juridicidad (la Justicia, el Bien Común, etc... ) y se ve obligado a apelar a un criterio subsidiario o ulterior iprecisamente respecto a los componentes básicos de mayor «jerarquía» del sistema!

La paradoja, sin embargo, podría pasarse por alto si fuese concebible que ambos criterios funcionaran simultáneamente. Este, sin embargo, no es el caso. Veamos por qué. Si el segundo criterio tiene como misión «identificar», «definir», o «constituir» la juridicidad de las normas justas, entonces parece lógicamente plausible, por lo que diré a continuación, que el candidato más idóneo para llevar a cabo esa tarea sean las «normas de competencia», es decir, aquellas normas secundarias que definen o cualifican como «autoridad» a ciertas agencias para emanar pautas y decisiones que, simplemente por su origen, se consideren «derecho». Pero, claro está, siendo un criterio prioritario el criterio moral de justicia, la actitud de los ciudadanos para identificar el derecho tendría que ser la de escrutar en las decisiones de la autoridad competente para ver si sus normas son justas, sólo en cuyo caso serían jurídicas y, por tanto, vinculantes. Como puede 
suponerse, una situación así no sólo pugna lógicamente, o hace irrelevante, la noción misma de «autoridad» sino que sería empíricamente inviable. La eficiencia de un sistema de normas de este tipo sería, seguramente, escasa.

Entonces el iusnaturalista puede dar un paso más allá y atribuir el valor moral que postula como inherente al concepto de derecho a las propias «normas de competencia» que le sirven para identificar a ese derecho. La «autoridad» constituida por esas normas sería así una autoridad «moral». Creo que merece la pena que exploremos un poco esta posibilidad.

Las normas de competencia o «power-conferring rules» se ajustan razonablemente bien a algunos de los rasgos lógicos que han venido apareciendo como propios de la cuestión de las relaciones entre moral y derecho. Y en particular se ajustan a la respuesta «metalingüística» y definicional que parece exigir dicha cuestión. De ahí su idoneidad como criterio subsidiario de identificación. En el pensamiento jurídico escandinavo se ha puesto especialmente de manifiesto esa doble condición. Von Wright, mejorando, a mi juicio, el análisis de Ross, establece con claridad la necesaria estratificación del lenguaje que esas normas implican al considerarlas «normas de orden superior» (von Wright, 1970). Y su carácter definicional ha sido establecido, por ejemplo, por Eckhoff y Sundby al considerar a las normas de competencia como «condiciones de validez» de normas jurídicas o como «normas cualificatorias», o, lo que es lo mismo, como expresiones que determinan qué actos y procedimientos deben ser considerados actos y procedimientos válidos para crear normas jurídicas. Las normas de competencia -se ha dicho- designan o definen qué agentes pueden producir efectos o normas jurídicas o cuando se produce la aparición de cualidades jurídicas (Hernández, 1984). Es muy importante hacer hincapié también en que estos autores ligan este tipo de normas con alguno de los sentidos de la noción de «validez», de forma que si pensamos que la «validez» es la propiedad identificatoria distintiva de las normas jurídicas, entonces ligar «normas de competencia» y «validez» equivale a atribuir a dichas normas la tarea de identificar a las reglas, actos o procedimientos como jurídicos. Ello sitúa a tales normas en un plano definicional que es extremadamente congruente con la naturaleza de la cuestión que nos ocupa, pues unas normas que «metalingüísticamente», definen la validez jurídica son seguramente el instrumento idóneo para responder a la pregunta de si, por definición, el derecho válido incorpora alguna propiedad moral.

Pues bien, el paso que ha de dar el iusnaturalista es el de 
atribuir una cierta validez o valor moral a las normas de competencia. Al hacerlo así, la noción de «validez jurídica», que en el pensamiento escandinavo tenía un significado predominante de «pertenencia», adquiere plenamente una dimensión de «vinculatoriedad» porque las normas de competencia adquieren la calidad de normas constitutivas de la moralidad del derecho, y la «autoridad» que esas normas crean deviene así una autoridad moral. El derecho válido, definido por su emanación de una autoridad moral, tiene así una conexión necesaria con la moral misma. Y las normas identificadas como jurídicas a través de las «power-conferring rules» pasan de ser consideradas como meros integrantes de un conjunto llamado «derecho» a ser consideradas también, en el sentido más estricto, razones para la acción. Son razones para la acción, no por su contenido, sino, por así decirlo, derivativamente, porque las normas de competencia a las que les ha sido atribuido un carácter moral constituyen por sí mismas razones para la acción que se proyectan sobre ellas.

Una manera útil de calibrar lo que ello significa puede ser tratar de establecer una taxonomía del funcionamiento de algunos de los diferentes tipos de razones para la acción prolongando tentantivamente un camino teórico ya abierto (Raz, 1975). En primer término podríamos hablar de aquellas razones comunes que la gente tiene y aduce para realizar acciones y tomar decisiones: placer, interés, deseo, utilidad, convicciones, afectos, etc... A la hora de actuar o decidir se realiza un balance de razones pro y razones contra de este tipo para llegar a un resultado. En segundo lugar se hallarían las normas de conducta, que según Raz, se presentan a sí mismas como razones de segundo orden cuya presencia «excluye» el balance de razones de primer orden mencionado. Si hay una norma de conducta, entonces el cálculo anterior queda desplazado en el sentido de que las preferencias y deseos, los intereses y las utilidades del agente no cuentan ya tanto como razones, al superponerse a ellos la norma de conducta como razón de orden superior para la acción.

Otro de mis interlocutores críticos (Juan Carlos Bayón) se muestra poco receptivo hacia esta idea de estratificación de las razones tal y como la presenta Raz. Alega, creo también que con razón, que por el mero hecho de que lo primero sea una simple vivencia individual (un deseo, por ejemplo) y lo segundo una norma vigente no puede aceptarse sin más que la norma se superponga como razón de orden superior a la vivencia individual. Si, por ejemplo, ese deseo individual pudiera ser calificado netamente cómo moral y la norma, en cambio, fuera meramente técnica, prudencial o de urbanidad, entonces no sería posible 
admitir sin más tal superposición. El mero hecho de que una norma exista (cualquier cosa que eso signifique) no puede ser interpretado sin más como que existe una razón excluyente que cancela el balance de razones de «primer orden». Ello dependería del carácter de estas razones. Pues bien, aceptando que las razones para la acción no son susceptibles de jerarquización según los criterios de Raz, sino únicamente por su calidad de prudenciales, morales, etc... creo, sin embargo, que la aportación de Raz puede seguramente mantenerse si se la utiliza dentro de cada una de esas «familias». Si yo tengo un interés prudencial en mi propia seguridad vial, por ejemplo, no es necesario que me plantee una estrategia individualizada de acción en cada cruce de calles cuando existe una norma prudencial de coordinación (semáforos, preferencias de paso, etc...). Simplemente sigo la norma, y en este supuesto la norma «excluye» el hipotético balance de razones prudenciales que yo pueda practicar en cada caso. Si, dentro de la «familia» de las razones morales, yo tuviera la convicción, por ejemplo, de que debo desarrollar comportamientos altruistas en beneficio del bienestar de mis conciudadanos, no tendría quizás que preguntarme en cada supuesto qué hacer si existiera una norma fiscal redistributiva eficaz. Simplemente con cumplir mis obligaciones con el fisco podría desentenderme del balance de razones morales que cada supuesto concreto suscitara. Y también en este sentido la norma excluye esa ponderación de razones. Lo importante de esta consideración es, precisamente, que pone de manifiesto que hay un cierto sentido correcto en la idea de Raz de que cuando una norma existe, o existe y tiene un contenido determinado, entonces no es necesario pararse a pensar. Y seguramente la fluidez de la convivencia y también quizás su eventual moralidad provienen seguramente de este mecanismo extraño que nos evita detenernos a pesar y contrapesar razones en cada situación característica.

Pero cuando establecemos estas interrelaciones entre razones individuales y normas estamos, creo, hablando exclusivamente de normas de conducta. Si avanzamos hasta incluir en el cuadro las normas de competencia las cosas se complican bastante. Porque las normas de competencia, o al menos las normas de competencia más importantes, operan atribuyendo autoridad a una agencia para que emita normas de conducta cuyo contenido, aunque pueda estar limitado en un marco amplio, es, por definición, abandonado de antemano. Es claro que si precisáramos exhaustivamente dicho contenido, la noción de autoridad y su existencia misma serían perfectamente prescindibles. Esto, que no es más que una descripción de lo que Hart llama «razones 
para la acción perentorias e independientes del contenido» (peremptory content-independent reasons) (Hart, 1982) configura una teoría de la autoridad de especial interés para nosotros. Porque si, dentro de una misma familia de razones, las normas de conducta operan ahorrándonos el pensar y razonar «de primer orden», las normas de competencia operarían haciendo lo mismo respecto de las normas de conducta. Todos aquellos razonamientos sobre la justificación interna de las normas de conducta quedarían cancelados mediante la apelación a una agencia de autoridad. Si no lo hicieran, cualquiera dudaría en considerar «autoridad» a esa agencia. La autoridad es tal porque sus decisiones suministran razones perentorias al margen del contenido de tales decisiones.

Pues bien, si esto es así el iusnaturalista que se ha visto obligado a conceder una dimensión moral a las normas de competencia se encuentra con un severo problema. Había planteado como exigencia para poder hablar de derecho el que las normas cumplieran con un criterio material de Justicia y como esto era insuficiente había añadido un criterio complementario de identificación de normas jurídicas a través de normas de competencia: serían jurídicas aquellas normas justas emanadas de una autoridad, pero no de una autoridad cualquiera, sino de una autoridad moralmente legítima. Y ahora vemos que sus dos criterios no pueden convivir porque las normas de competencia como razones morales «independientes del contenido» $\mathrm{y}$ «perentorias» tienden a ignorar precisamente la consideración de la justicia del contenido de las normas de conducta. El criterio material de justicia se ha esfumado y el iusnaturalista se encuentra con que para mantener su teoría de la conexión necesaria entre derecho y moral tiene que hacer descansar la moralidad del derecho en la autoridad que lo crea, y nada más. El derecho sería entonces moral porque emana de una autoridad moral, cualquiera que sea el contenido de sus normas. Y esto hace desembocar al iusnaturalismo en una ética del tipo «mandato divino», es decir, le hace desembocar en algo incompatible con una ética ilustrada o crítica: en la idea de que la autoridad como razón moral perentoria nos ahorra la práctica del debate moral sobre el contenido de las normas.

En definitiva, si se asume que no es posible identificar el derecho o las normas jurídicas sin alguna noción de normas de competencia, y se pretende después que hay una conexión conceptual entre derecho y moral la existencia del derecho y la existencia de una moralidad crítica o ilustrada empieza a ser incompatibles. Si llamamos «crítica», «ilustrada», «esclarecida», etc... 
a una actitud moral que postula que las reglas y valores éticos emergen de una reflexión individual o colectiva de agentes libres y racionales sobre las pautas de acción y decisión, entonces estos iusnaturalistas no pueden pretender participar de una teoría moral de este tipo. Si la identificación del derecho válido se ha de llevar a cabo a través de la idea de «autoridad» y se colorea la naturaleza de esa autoridad con tintes inequívocamente morales, entonces dicha autoridad se presenta emitiendo decisiones y normas cuyo contenido queda excluido de toda argumentación moral: es decir, se trata de una autoridad cuya mera existencia trata de establecer una restricción «a priori» a la discusión racional del contenido de las normas o supone que tal restricción es necesaria para la existencia misma del derecho. Tal consecuencia me parece inaceptable, porque creo que la existencia del derecho y la extensión de la actitud moral crítica no son fenómenos que se excluyan entre sí.

En suma, por todas las razones que he alegado, reivindico el punto de vista del observador crítico para enfrentar el problema práctico fundamental que se esconde en la centenaria polémica de las relaciones entre derecho y moral; y desde ese punto de vista el enunciado «Esto es derecho válido pero es inmoral» no tiene tacha alguna de contradictorio porque, desde él, no hay conexión conceptual entre derecho y moral. Pero el iusnaturalista que, como observador externo (que es lo único que puede ser un iusnaturalista consecuente), niega esa conclusión entra de lleno en un camino que le conduce a una aporía, ya que su propuesta de comprender el derecho «moralmente» se salda con la necesidad de aceptar una moralidad incomprensible.

\section{BIBLIOGRAFÍA}

Alexy, Robert, «On necessary Relations Between Law and Morality» Ratio Juris, vol. 2, núm. 2, julio 1989.

Bulygin, Eugenio, «Sobre la regla de reconocimiento», en Derecho, Filosofía y Lenguaje. Homenaje a Ambrosio L. Gioja, Ed. Astrea, Buenos Aires, 1976.

Castiglione, Silvana, «Punto di vista interno ed efficacia», en La teoria generale del diritto. Studi dedicali a Norberto Bobbio, a cura di Uberto Scarpelli, Ed. di Comunitá, Milán, 1983. 
Hart, Herbert L. A., Essays on Bentham, Clarendon Press, Oxford, 1982.

Hernández Marín, Rafael, El Derecho como dogma, Tecnos, Madrid, 1984.

Laporta, Francisco J., «Über die Beziehungen zwischen Recht und Moral», en Spanische Studien zur Rechtstheorie und Rechisphilosophie, Berlín, 1990.

MacCormick, Neil, «Comment» a G. J. Posterna, en Issues in Contemporary Legal Philosophy, Ed. by Ruth Gavison, Clarendon Press, Oxford, 1987.

Nino, Carlos, El constructivismo ético, CEC, Madrid, 1989.

Raz, Joseph, The Authority of Law, Clarendon Press, Oxford, 1979.

—, Practical Reason and Norms, Hutchinson, Londres, 1975 (2. ${ }^{\text {a }}$ edición, Princeton University Press, 1990).

Von Wright, G. Henrik, Norma y acción. Una investigación lógica. Ed. Tecnos, Madrid, 1970 . 